Relations industrielles

Industrial Relations

\title{
Le coût des soins médicaux, Bureau International du Travail, Genève, 1959, 260 pp.
}

\section{Émile Gosselin}

Volume 14, numéro 4, octobre 1959

URI : https://id.erudit.org/iderudit/1022139ar

DOI : https://doi.org/10.7202/1022139ar

Aller au sommaire du numéro

Éditeur(s)

Département des relations industrielles de l'Université Laval

ISSN

0034-379X (imprimé)

1703-8138 (numérique)

Découvrir la revue

Citer ce compte rendu

Gosselin, É. (1959). Compte rendu de [Le coût des soins médicaux, Bureau International du Travail, Genève, 1959, 260 pp.] Relations industrielles / Industrial Relations, 14(4), 620-621. https://doi.org/10.7202/1022139ar

Tous droits réservés @ C Département des relations industrielles de l’Université Laval, 1959
Ce document est protégé par la loi sur le droit d'auteur. L’utilisation des services d'Érudit (y compris la reproduction) est assujettie à sa politique d'utilisation que vous pouvez consulter en ligne.

https://apropos.erudit.org/fr/usagers/politique-dutilisation/ 
Le lecteur trouvera dans l'introduction à quels critères doit répondre un système pour pouvoir être considéré comme faisant partie du système national de sécurité sociale. Dans une première partie, l'étude présente, pour 32 pays, les tableaux de base indiquant les divers postes de recettes et de dépenses.

Dans une deuxième partie on essaie d'établir, à l'aide de tableaux, la comparaison internationale des données de la sécurité sociale. La troisième partie comporte une série très précieuse de tableaux sur diverses formes de sécurité sociale propres à divers pays.

Ce recueil doit faire partie de la bibliothèque de tous ceux que leur activité économique ou leur situation sociale obligent à s'occuper de l'élaboration ou de la mise en application de systèmes de sécurité sociale publics ou privés. Economistes, sociologues, spécialistes du travail y trouveront également une source inestimable de renseignements rigoureusement controlés et à date.

EMme Gosseln

Le cô̂t des soins médicaux, Bureau International du Travail, Genève, 1959, $260 \mathrm{pp}$.

Dans l'étude intitulée Le coût des soins médicaux », travail présenté avec extrêmement de minutie, le Bureau International du Travail a réuni une forte documentation relative aux différents soins médicaux et à leur coût total dans quatorze pays qui possiecient des services de soins médicaux étendus. Une attention spéciale est portée à la situation aux Etats-Unis. Les statistiques à ce sujet ont été ondonnés de façon à permettre au lecteur de comparer les coûts non seulement à l'intérieur d'un même pays mais d'un pays à l'autre.

Le volume comporte cinq chapitres et plusieurs annexes où l'on donne le détail des calculs par pays, la répartition des dépenses publiques et privées et l'affectation de ces dépenses selon les services rendus, ainsi qu'une série de tableaux comparatifs indiquant le nombre de personnes protégées pour chacun des régimes de sécurité sociale et les dépenses pour prestations médicales par personne protégée.

Dans le ler chapitre de l'ouvrage, l'on entend préciser l'étendue de la protection médicale en couvrant deux aspects: le nombre et les catégories de personnes protégées selon chaque pays, les risques ou éventualités couverts par les différents systèmes, et les répercussions que peuvent représenter ces deux facteurs si on les évalue différemment d'un pays à l'autre sur la comparabilité des dépenses pour prestations médicales. Nous recommandons très particulièrement $\propto$ chapître à ceux qui ont à établir ou à négocier des plans à l'intention de catégories de personnes bien définies.

Dans le deuxième chapître on évalue pour chaque pays la dépense pour diverses prestations médicales ainsi que le coût des soins médicaux reçus par les personnes protégées par opposition aux dépenses des institutions de sécurité sociale pour ces mêmes soins.

Dans le troisième chapître, l'on tente de dégager pour une période donné, l'évolution des dépenses pour prestations médicales selon les différents régimes de sécurité sociale offrant une protection médicale quelconque et de situer chaque catégorie de soins par rapport aux autres au point de vue coût.

Le chapitre IV analyse le revenu que les médecins, et dentistes tirent de la sécurité sociale et d'autres sources. Les conclusions de ce chapître en regard des chapîtres précédents s'avéreraient très utiles, en particulier pour les employeurs et les associations professionnelles qui ont à négocier en vue d'établir les barêmes de rémunération pour les personnes affectées aux soins médicaux protégés par les régimes de sécurité sociale.

Enfin le chapitre V tire les conclusions de l'analyse faite au cours des chapitres précédents. On notera en particulier dans quelle mesure le coût des divers soins médicaux affecte le revenu moyen, à quoi et dans quelle proportion sont affectées les diverses dépenses: hospitalisation, médecins, spécialistes, remèdes, dentistes. Quant à la tendance des dépenses, il faut souligner que sauf en France et en 
Italie, pour une période de 10 ans allant de 1945 à 1955 , «les dépenses des régimes de sécurité sociale par personne protégées, exprimées en pourcentage du revenu national ou du salaire de référence, sont restées relativement stables ». L'étude s'insorit en faux "contre l'opinion très répandue selon laquelle il existe une tendance générale à l'augmentation du coût des soins médicaux; en tout cas, cette augmentation ne dépasserait pas l'augmentation du revenu national et du salaire de ré férence, bien que le coût des soins hospitaliers semble en prendre le chemin $\gg$. (p. 191-192) Egalement, le BIT note à la page 188 une constatation qui nous paraît assez troublante:

- Il semble que ce soit aux différences dans le coût des produits pharmaceutiques et dans celui des soins de spécialistes qu'il faut imputer les écarts les plus importants dans le coût global par rapport au revenu moyen par personne active, tels que les fait ressortir cette étude. Il se peut que des traditions médicales différentes entraînent des préférences pour certaines catégories de soins. Il semble exister une certaine corrélation entre le volume des soins dispensés par les spécialistes et la consommation de fournitures pharmaceutiques, mais les différences dans la définition des fournitures pharmaceutiques peuvent avoir une incidence indéniable sur la comparabilité de ces données. 》

\section{Emile Gosselin}

\section{American Labor's Role in Less Deve-} lopped Countries. A report on a conference held at Cornell University October 12-17 1958. New-York School of Industrial and Labor Relations, Cornell University, 70 pp.

L'attitude d'un pays comme les EtatsUnis envers les pays moins développés est de nature à avoir des répercussions considérables sur l'évolution des destinées du monde. L'industrialisation amène les masses laborieuses à s'organiser. Le mouvement ouvrier américain est donc vitalement intéressé à $\mathrm{y}$ jouer un rôle autant que les capitalistes et le «State Department \$. En effet, depuis la fin de la guerre partidulièrement, les syndicats américains ont été invités à fournir du personnel soit dans le service diplomatique soit dans les agences d'assistance aux pays moins développés soit enfin dans les organismes internationaux du travail. Le but de ce congrès, organisé conjointement par le Département des affaires étrangères de l'AFL-CIO et l'Ecole des relations industrielles et du travail de l'Etat de New York, était d'examiner certains problèmes qui pouvaient affecter l'activité syndicale sur le plan international.

Cet ouvrage contient le texte des exposés ainsi qu'un résumé des discussions qui suivirent. La simple nomenclature des problèmes abordés indique déjà l'intérêt que peut apporter un tel ouvrage:

La politique et les programmes des Etats-Unis dans les pays moins développés; le rôle du syndicalisme libre dans les pays moins développés; L'aide et le commerce en regard de l'économie américaine; Les agences internationales du travail (la Confédération internationale des syndicats libres; l'organisation internationale du travail, les secrétariats professionnels internationaux); Les besoins éducatifs des syndicats libres dans les pays moins développés; Jusqu'à quel point les syndicats américains peuvent aider le développement des syndicats libres dans les pays sous-développés?; Problèmes de direction des entreprises dans lev pays moins développés; un cas: l'organisation syndicale et les relations du travail en Indonésie.

Evidemment, on ne pouvait traiter à fond chacun de ces sujets. Mais les questions soulevées tant par les conférenciers que par l'assistance, même si parfois elles restent sans solution précise, montrent la complexité des problèmes et l'urgence d'y porter une attention sérieuse.

G. D.

The American Labor Movement. Edited by Walter M. Daniels. - The Reference Shelf, Vol. 30, No. 3. - M. W. Wilson Company, New York, 1958,225 pp. $\$ 2.00$.

Le syndicalisme ouvrier est une institution dynamique dont il est difficile de saisir tous les aspects dans son évolution constante. 